Supporting Information

\title{
Functional Integration of Catalysts with Si Nanowire Photocathodes for Efficient Utilization of Photogenerated Charge Carriers
}

\author{
Sung Yul Lim, ${ }^{* \dagger}$ Daye Seo,${ }^{\ddagger}$ Min Seok Jang, ${ }^{\S}$ and Taek Dong Chung $*, \star \star$
}

\footnotetext{
${ }^{\dagger}$ Department of Chemistry and Research Institute for Basic Science, Kyung Hee University, Seoul 02447, Korea

Department of Chemistry, Seoul National University, Seoul 08826, Korea

${ }^{\S}$ School of Electrical Engineering, Korea Advanced Institute Science and Technology (KAIST), Daejeon 34141, Korea
}

* Corresponding author. E-mail: limsy @khu.ac.kr (S.Y.L.); tdchung@ snu.ac.kr (T.D.C.) 


\section{EXPERIMENTAL METHODS}

\subsection{Photoelectrochemical measurements}

To generate simulated AM 1.5G irradiation, a $150 \mathrm{~W}$ xenon arc lamp was utilized with an air mass filter (1.5 Global), and the intensity was set to $100 \mathrm{~mW} \mathrm{~cm}^{-2}$ by adjusting the distance between the light source and the photoelectrodes. The light intensity was calibrated using a radiometer (Solar Light, PMA-2100) and a pyranometer (Solar Light, PMA-2144). For all the electrochemical experiments, an $\mathrm{Ag} / \mathrm{AgCl}$ electrode (3 $\mathrm{M} \mathrm{NaCl}$, Bioanalytical System) and a coiled Pt wire were employed as the reference and counter electrodes, respectively. The potential from the $\mathrm{Ag} / \mathrm{AgCl}$ reference electrode was calibrated for conversion to the reversible hydrogen electrode (RHE) scale by measuring the stabilized open-circuit potential versus a commercial RHE electrode (ALS Co.) in an $\mathrm{H}_{2}$-purged $0.5 \mathrm{M} \mathrm{H}_{2} \mathrm{SO}_{4}$ electrolyte. The cyclic voltammograms were acquired under forced convective conditions with $300 \mathrm{rpm}$ of stirring.

\subsection{Physical characterization}

The scanning electron microscope (SEM) was used with a SUPRA 55VP (Carl Zeiss) at 2 $\mathrm{kV}$ as an accelerating voltage at the National Instrumentation Center for Environmental Management of Seoul National University (SNU). To observe the cross-sectional view of the SiNW/AgPt by transmission electron microscope (TEM), the sample was prepared by a focused ion beam SMI3050SE (SII Nanotechnology). The TEM analysis was conducted with a JEM-300F (JEOL) at $300 \mathrm{kV}$ at the Research Institute of Advanced Materials in SNU. To quantify the Pt loading by utilizing the inductively coupled plasma-mass spectrometry (ICP-MS, nexION 350D, PerkinElmer Inc., $)$, the photocathodes were immersed in $5 \mathrm{~mL}$ of aqua regia $\left(3: 1 \mathrm{HCl}: \mathrm{HNO}_{3}\right)$ for 
overnight to dissolve Pt and $\mathrm{Ag}$ into the solutions. Then, $5 \mathrm{~mL}$ of DI water was added and proceeded the analysis at the National Center for Inter-University Research Facility in SNU. The absorption spectra was acquired with a Cary5000 (Agilent) at Yonsei Center for Research Facilities. 


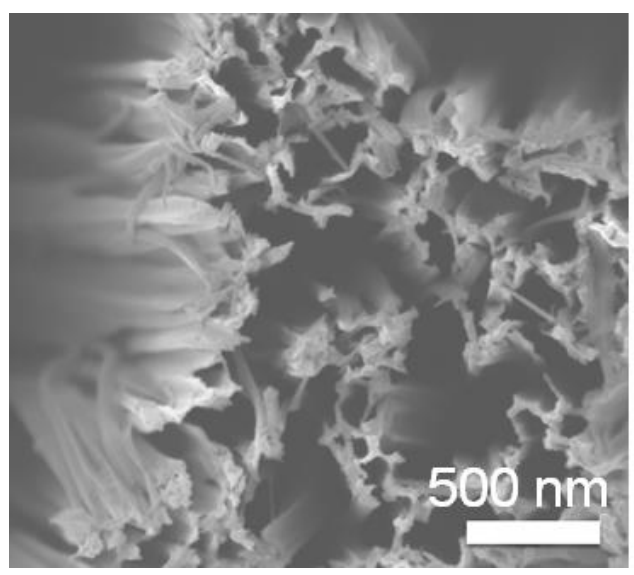

Figure S1. SEM image of bare SiNW. 
(a)

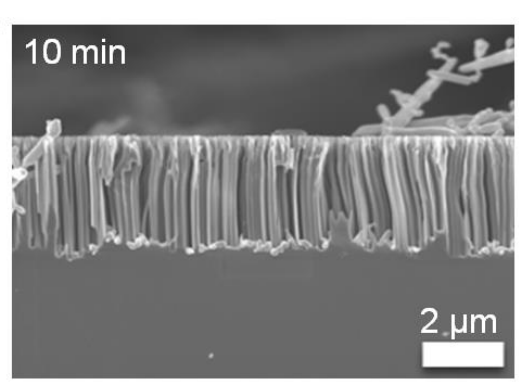

(c)

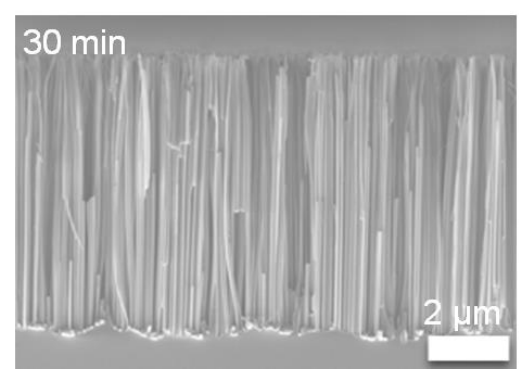

(b)

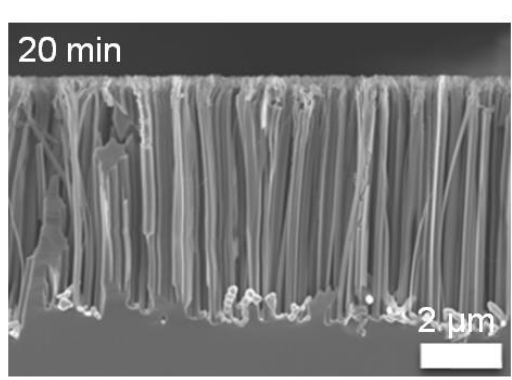

(d)

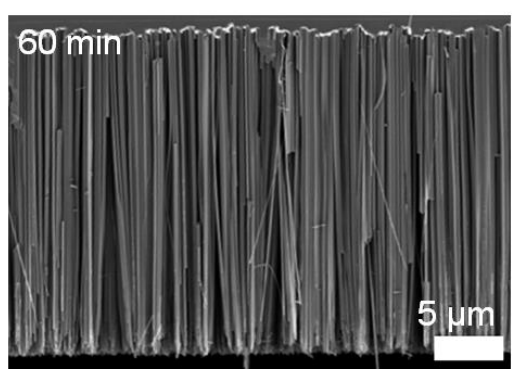

Figure S2. Cross-sectional SEM images of SiNW/AgPt photocathodes with different SiNW lengths fabricated with etching times of (a) 10, (b) 20, (c) 30, and (d) 60 min. 


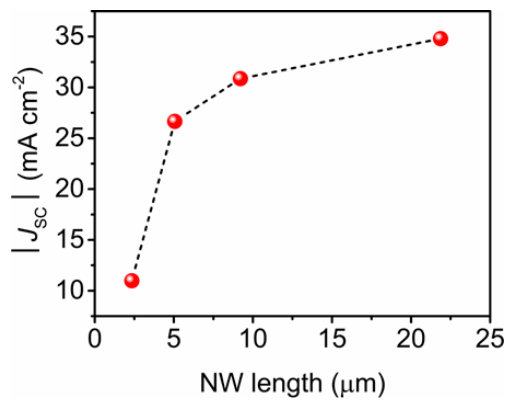

Figure S3. Effect of length of the SiNWs on saturated photocurrent density. 
Table S1. Potential for acquiring $-10 \mathrm{~mA} \mathrm{~cm}^{-2}\left(V_{@-10 \mathrm{~mA} \mathrm{~cm}-2}\right)$, current density at $0 \mathrm{~V}_{\mathrm{RHE}}\left(J_{@ 0} \mathrm{~V}_{\mathrm{RHE}}\right)$, $J_{\mathrm{SC}}$, and $V_{\mathrm{OC}}$ in voltammograms for HER of SiNW-based photocathodes under AM 1.5G illumination.

\begin{tabular}{|c|c|c|c|c|}
\hline $\begin{array}{c}\text { SiNW-based } \\
\text { photocathodes }\end{array}$ & $\begin{array}{c}V_{@-10 \mathrm{~mA} \mathrm{~cm}^{-2}} \\
\left(\mathrm{~V}_{\mathrm{RHE}}\right)\end{array}$ & $\begin{array}{c}J_{@ 0} \mathrm{~V}_{\mathrm{RHE}} \\
\left(\mathrm{mA} \mathrm{cm}^{-2}\right)\end{array}$ & $\begin{array}{c}J_{\mathrm{SC}} \\
\left(\mathrm{mA} \mathrm{cm}^{-2}\right)\end{array}$ & $V_{\mathrm{OC}}$ \\
\hline $\mathrm{Pt} / \mathrm{C}$ & -0.0179 & 0 & $\mathrm{~N} / \mathrm{A}$ & 0 \\
\hline SiNW/Ag & -0.776 & -0.0717 & $\mathrm{~N} / \mathrm{A}$ & 0.336 \\
\hline $\begin{array}{c}\text { SiNW/Ag-Pt } \\
\left(\begin{array}{c}0 \text { min etching for } \\
\text { NW formation })\end{array}\right.\end{array}$ & -0.298 & -4.63 & -10.4 & 0.288 \\
\hline $\begin{array}{c}\text { SiNW/Ag-Pt } \\
\left(\begin{array}{c}20 \text { min etching for } \\
\text { NW formation })\end{array}\right.\end{array}$ & -0.0169 & -8.95 & -26.1 & 0.207 \\
\hline $\begin{array}{c}\text { SiNW/Ag-Pt } \\
(30 \text { min etching for } \\
\text { NW formation })\end{array}$ & 0.0194 & -11.7 & -30.6 & 0.250 \\
\hline $\begin{array}{c}\text { SiNW/Ag-Pt } \\
(60 \text { min etching for } \\
\text { NW formation })\end{array}$ & -0.0189 & -8.59 & -35.0 & 0.221 \\
\hline Pts/SiNW & -0.0166 & -9.09 & -24.8 & 0.350 \\
\hline Pt $/$ SiNW & 0.217 & -22.5 & -22.8 & 0.344 \\
\hline $\mathrm{Pt}_{\mathrm{S}} / \mathrm{SiNW} / \mathrm{Ag}-\mathrm{Pt}$ & 0.182 & -22.0 & -25.1 & 0.345 \\
\hline
\end{tabular}

